\title{
A High-Resolution RNA Expression Atlas of Retinitis Pigmentosa Genes in Human and Mouse Retinas
}

\author{
Dragana Trifunovic, ${ }^{1}$ Marianthi Karali, ${ }^{1}$ Davide Camposampiero, ${ }^{2}$ Diego Ponzin, ${ }^{2}$ \\ Sandro Banfi, ${ }^{* 1}$ and Valeria Marigo*,3
}

Purpose. Retinitis pigmentosa (RP) is one of the leading causes of visual handicap in the world population and is characterized by high genetic heterogeneity. The study of the disease mechanisms and the development of efficient therapeutic approaches have mostly relied on the availability of animal models for this condition, so far. Nevertheless, little information is available about the RNA expression profiles of RP genes in the human retina. An expression atlas of 34 known RP genes in human and murine retinas was generated to overcome this lack of information.

Methods. Appropriate templates were retrieved for 34 RP genes that were used to perform RNA in situ hybridization studies on human and murine adult eyes.

Results. Most of the genes displayed similar patterns between human and mouse retina. Different expression patterns were observed for the CNGB1, USH2A, and FSCN2 genes, compared with those in previously reported profiles. In addition, different expression profiles were detected for the RPGR, CA4, $P A P 1, R G R$, and $R L B P 1$ genes in human and mouse retinas.

Conclusions. The first gene expression atlas has been generated of RP genes in human and murine retinas. Differences observed in the expression patterns of some genes in humans and mice, will open new perspectives on the function of these genes and their putative roles in disease pathogenesis. (Invest Ophthalmol Vis Sci. 2008;49:2330-2336) DOI:10.1167/iovs.071513

$\mathbf{R}$ etinitis pigmentosa (RP) is one of the leading causes of inherited visual handicap in the world population, with a prevalence of 1 in $3500 .{ }^{1}$ A remarkable feature of the disorder is its high genetic heterogeneity. So far, more than 50 genes responsible for nonsyndromic forms have been mapped (RetNet; http://www.sph.uth.tmc.edu/Retnet/ provided in the

From the ${ }^{1}$ Telethon Institute of Genetics and Medicine (TIGEM), Naples, Italy; the ${ }^{2}$ Fondazione Banca degli Occhi del Veneto, Venice, Italy; and the ${ }^{3}$ Department of Biomedical Sciences, University of Modena and Reggio Emilia, Modena, Italy.

Supported by European Union Grants MRTN-CT-2003-504003 and LSHG-CT-2005-512036; the Italian Telethon Foundation; European Community Grant MRTN-CT-2003-504003 (DT); Research Grants GGP06096 from Fondazione Telethon (VM), PRIN 2006053302_003 (VM), and NEI Grant 1R01EY015136-01 (VM).

Submitted for publication November 26, 2007; revised January 18, 2008; accepted April 14, 2008.

Disclosure: D. Trifunović, None; M. Karali, None; D. Camposampiero, None; D. Ponzin, None; S. Banfi, None; V. Marigo, None

The publication costs of this article were defrayed in part by page charge payment. This article must therefore be marked "advertisement" in accordance with 18 U.S.C. $\$ 1734$ solely to indicate this fact.

*Each of the following is a corresponding author: Sandro Banfi, Telethon Institute of Genetics and Medicine, Via P. Castellino 111, 80131 Naples, Italy; banfi@tigem.it.

Valeria Marigo, Department of Biomedical Sciences, University of Modena and Reggio Emilia, Via G. Campi 287, 41100 Modena, Italy; valeria.marigo@unimore.it. public domain by the University of Texas Health Science Center, Houston, TX). RP can be inherited in all the main modes of inheritance (i.e., autosomal dominant, autosomal recessive, $\mathrm{X}$-linked, and mitochondrial). ${ }^{2,3}$ Nevertheless, most cases are apparently sporadic. Genes responsible for RP are generally expressed at significant levels in the cells of the neural retina or in the retinal pigment epithelium and can also be classified, based on their predicted functional activities, into the following groups: members of the phototransduction cascade and of the visual cycle pathway, transcription factors, splicing factors, photoreceptor structural proteins, genes involved in protein folding or trafficking and $\mathrm{pH}$ balance. Numerous animal models have been generated to gain a better understanding of the genetic basis and molecular mechanisms of retinal degeneration and to develop therapeutic strategies. ${ }^{4}$ Most of the animal models used in eye research are murine models, mainly because mice are easily handled, have a relatively short lifespan, and have genes that usually share a high percentage of sequence identity with the corresponding human genes. Nonetheless, some notable differences between the human and the murine retina may hamper the transfer of the knowledge gained from mouse models to patients. For example, mice, like most nocturnal animals, do not have cone-rich areas for visual acuity, such as the fovea. ${ }^{5}$ Thus, differences in distribution of rods and cones in human and mouse retinas may be an obstacle in transferring observations derived from mouse models to humans. In addition, only two types of cones, short- and middle-wavelength, are present in the murine retina, whereas the human retina also contains long-wavelength cones. More important, not all mouse models show the same phenotype as patients with the same gene lesion. For example, patients with dominant RP or Leber congenital amaurosis, caused by mutations in the IMPDH1 gene, show a more severe disorder than is seen in a mouse model with a mutation in the orthologous murine gene. ${ }^{6}$ Hence, to design efficient therapeutic approaches for RP, it is essential to have detailed information about the expression of RP genes in the human retina. Unfortunately, to date, little information is available about the expression patterns of RP genes at the cellular level in the human retina, and all the current knowledge is inferred through studies in the mouse. To overcome this lack of information, we have generated an expression atlas by RNA in situ hybridization (ISH) of all the genes responsible for RP that have been identified so far. In parallel we performed expression studies of the corresponding murine orthologues and compared the RNA expression patterns between the two species.

\section{Materials ANd Methods}

\section{RNA ISH on Cryosections}

Antisense probes and sense control probes for RNA ISH experiments were obtained by using a variety of approaches, including public expressed sequence tag (EST) clones, PCR amplification of human/ mouse genomic DNA or cDNA prepared from human/mouse total retinal RNA with the specific primers tailed by sequences recognized by the RNA polymerases (T3, T7, or SP6). Finally, some of them were 
TABLE 1. Information about Donor Eyes

\begin{tabular}{lllr}
\hline Individual & Age & Cause of Death & $\begin{array}{c}\text { Postmortem Time } \\
\text { before Eye Removal } \\
\text { (Hours:Minutes) }\end{array}$ \\
\hline $43427 / 43428$ & 30 & Trauma & $19: 45$ \\
$50608 / 50609$ & 48 & Tumor & $6: 45$ \\
52493 & 37 & Trauma & $5: 45$ \\
53041 & 48 & Cardiovascular & $5: 45$ \\
60933 & 48 & Tumor & $5: 10$ \\
60935 & 41 & Cardiovascular & $8: 45$ \\
62135 & 58 & Cardiovascular & $22: 15$ \\
$62658 / 62659$ & 56 & Tumor & $19: 40$ \\
\hline
\end{tabular}

generous gifts of external investigators. For more detailed information about each template, please refer to the online database (http:// www.tigem.it/RPexp/).

Human eye bulbs were obtained from eight cornea donors (Table 1) collected by the Italian Eye Bank (Fondazione Banca degli Occhi del Veneto, Venice, Italy). The research followed the tenets of the Declaration of Helsinki.

After removal of the lens, eye bulbs were fixed for 48 hours in $4 \%$ paraformaldehyde (PFA) in phosphate-buffered saline (PBS), followed by cryoprotective treatment with $30 \%$ sucrose in PBS and embedding in $7.5 \%$ gelatin. Eyes from 3 month old CD1 mice were treated in the same way. Twenty-micrometer cryosections were collected on slides (Superfrost Plus; Fisher Scientific, Pittsburgh, PA), air dried, and used for RNA ISH experiments, as previously described. ${ }^{7,8}$ Briefly, cryosections were fixed in $4 \%$ paraformaldehyde (PFA) in PBS for 15 minutes. After being bleached with $6 \% \mathrm{H}_{2} \mathrm{O}_{2}$ in PBS with $0.1 \%$ Tween 20 , the sections were treated with either 1 (mouse tissue) or 10 (human tissue) $\mu \mathrm{g} / \mathrm{mL}$ proteinase $\mathrm{K}$ for 15 minutes, followed by postfixation with $4 \%$ PFA and $0.2 \%$ glutaraldehyde. Hybridization with digoxigenin-labeled probes $(2 \mu \mathrm{g} / \mathrm{mL})$ was performed overnight at appropriate temperatures for each probe, as listed in the database. The hybridized sections were washed with $50 \%$ formamide, $4 \times$ SSC, and $1 \%$ SDS at hybridization temperature and with $50 \%$ formamide and $2 \times$ SSC at temperature $5^{\circ} \mathrm{C}$ below the hybridization temperature. Sections were blocked for 1 hour with $1 \%$ blocking reagent (Roche Diagnostics, Milan, Italy) in MABT (100 mM maleic acid, $150 \mathrm{mM} \mathrm{NaCl}, 0.1 \%$ Tween 20; pH 7.5), containing $10 \%$ sheep serum, and incubated with alkaline phosphatase (AP)-labeled anti-digoxigenin antibody (1:2000; Roche) in MABT with $1 \%$ blocking reagent overnight at $4^{\circ} \mathrm{C}$. After extensive washes with Tris-buffered saline containing $0.1 \%$ Tween 20 (TBST), the sections were exposed to the solution at $\mathrm{pH} 9.5$ containing the substrate for AP, nitroblue tetrazolium and 5-bromo-4-chloro-3-indoyl phosphate (NBTBCIP; Sigma-Aldrich, Milan, Italy). Reaction was blocked by washes with PBS at $\mathrm{pH} 5.5$, followed by postfixation in $4 \%$ PFA for 20 minutes. The slides were coverslipped with $70 \%$ glycerol in PBS or dehydrated and mounted (Eukitt mounting medium; Sigma-Aldrich). The authors confirm adherence to the ARVO Statement for the Use of Animals in Ophthalmic and Vision Research.

\section{Reverse Transcription-Polymerase Chain Reaction}

RT-PCR was performed on CDNA derived from total RNA purified from adult eyes of either wild-type mice or mice lacking photoreceptors (homozygous Aipl1 knockout animals, ${ }^{9} \mathrm{Aipl1}^{-/-}$, kindly provided by Michael Dyer (St. Jude Children's Research Hospital, Memphis, TN). $\mathrm{Nrl}$ amplification was performed with two sets of oligonucleotide primers, the first spanning the first and second exons (Nrl-1: forward 5'-AGCCGTCTGGGAATGAGCGA-3' and reverse 5'-GTGATGATGTAATGGCAGAGA-3'), and the second pair spanning the second and third exons (Nrl-2: forward 5'-GGTTCTCGGGCTGAGTCCCG-3' and reverse 5'-CGCAGCCCCGCAGCTGCCGG-3'). RT-PCR for Cngb1, Ush2a, and Fscn 2 was performed with the following specific oligonucleotide primers: Cngb1: forward 5'-CCCACCTGAGAGCCAGGCTC-3', reverse 5'-
CCCCCGTGCTTTCCTCGGGC-3'; Ush2a: forward 5'-TTCTGTCCTTGATTCTACAA-3', reverse 5'-CAGACACACTTTGGCTGCTC- 3 '; and Fscn2: forward 5'-TGGGTCATGAGACATTCCTG-3', reverse 5'-CTGCCCGTTTTTCTTCATGC-3'.

\section{Results \\ Expression Atlas of RP Genes}

To generate an expression atlas representing the RP genes, we retrieved appropriate human and murine templates for 34 known genes involved in the pathogenesis of nonsyndromic forms of RP (Table 2).

Spatial distributions of the selected transcripts were analyzed by RNA ISH experiments both in adult human and murine eye sections. For the human transcripts, all the experiments were performed on eye sections obtained from more than one individual. To overcome possible differences due to heterogeneity in human samples, we report only expression patterns confirmed in at least two individuals. Of the 34 analyzed genes by RNA ISH, only one (MERTK) failed to show a signal both in human and mouse eye sections, even though RT-PCR experiments confirmed the expression of MERTK in both human and mouse eyes (data not shown). Most (29/34) of the analyzed genes showed identical expression patterns in human and mouse eyes (Table 2). Examples of similar expres-

TABLE 2. Summary of the RNA In Situ Hybridization Expressions in Human and Mouse Retinas

\begin{tabular}{|c|c|c|}
\hline Gene & $\begin{array}{l}\text { RNA Localization } \\
\text { in Human }\end{array}$ & $\begin{array}{l}\text { RNA Localization } \\
\text { in Mouse }\end{array}$ \\
\hline$A B C A 4$ & PR & PR \\
\hline AIPL1 & PR & PR \\
\hline$C A 4$ & PR, INL, GCL & ND \\
\hline CERKL & PR, INL, GCL & PR, INL, GCL \\
\hline CNGA1 & PR & PR \\
\hline CNGB1 & PR, INL, GCL & PR, INL, GCL \\
\hline CRB1 & PR, INL & PR, INL \\
\hline$C R X$ & PR, INL & PR, INL \\
\hline FSCN2 & PR, INL, GCL & PR, INL, GCL \\
\hline GUCA1B & PR & PR \\
\hline IMPDH1 & PR, INL, GCL & PR, INL, GCL \\
\hline$L R A T$ & RPE & RPE \\
\hline MERTK & ND & ND \\
\hline$N R 2 E 3$ & PR & PR \\
\hline$N R L$ & PR, INL, GCL & PR, INL, GCL \\
\hline$P A P I$ & PR, INL, GCL & PR, INL, GCL \\
\hline PDE $6 A$ & PR & PR \\
\hline PDE6B & PR & PR \\
\hline$P R P F 3$ & PR, INL, GCL & PR, INL, GCL \\
\hline PRPF3I & PR, INL, GCL & PR, INL, GCL \\
\hline PRPF8 & PR, INL, GCL & PR, INL, GCL \\
\hline RDH12 & PR & PR \\
\hline$R D S$ & PR & PR \\
\hline$R G R$ & RPE, PR, INL & RPE \\
\hline $\mathrm{RHO}$ & PR & PR \\
\hline$R L B P I$ & RPE, PR, INL, GCL & RPE, INL, GCL \\
\hline$R P I$ & PR & PR \\
\hline$R P 2$ & PR, INL, GCL & PR, INL, GCL \\
\hline RPE65 & RPE & RPE \\
\hline$R P G R$ & PR, INL & PR \\
\hline RPGR-ORF15 & PR & PR, INL, GCL \\
\hline$R P G R I P$ & PR & PR \\
\hline$S A G$ & PR & PR \\
\hline TULP1 & PR, INL, GCL & PR, INL, GCL \\
\hline USH $2 A$ & PR, INL, GCL & PR, INL, GCL \\
\hline
\end{tabular}

PR, photoreceptors; INL, inner nuclear layer containing bipolar cells, horizontal cells, amacrine cells, Müller glia cells; GCL, ganglion cell layer; RPE, retinal pigment epithelium, ND, not detectable. 




Figure 1. Examples of genes with similar expression patterns in human and murine eyes. RNA expression patterns of the IMPDH1 (A, E), NR2E3 (B, F), PRPF31 (C, G), and $R D S(\mathbf{D}, \mathbf{H})$ genes in human $(\mathbf{A}-\mathbf{D})$ and mouse (E-H) adult retina sections. The strong signal in the inner segment of photoreceptors (IS) reflects the distribution of the cytoplasmic space where RNA accumulates in photoreceptors. ONL, outer nuclear layer. sion patterns are shown in Figure 1 . The reproducibility of the results demonstrates that we successfully optimized RNA ISH experiments on human adult eye sections, and that the obtained expression profiles are reliable. All the expression data generated during this work have been collected into the RP gene expression atlas database and can be accessed at http:// www.tigem.it/RPexp/ (provided in the public domain by the Telethon Institute of Genetics and Medicine [TIGEM], Naples, Italy). The database also contains data obtained with the sense control probes for all the genes analyzed and information on the eye samples used in the study for each analyzed gene, including donor age, cause of death, and postmortem time.

\section{Expression of CNGB1, USH2A, and FSCN2 in the Retinal Cell Layers}

Among the 29 genes showing identical expression patterns in human and murine retinas, we observed interesting RNA localization for the following genes: CNGB1, USH $2 A$, and FSCN2 Our expression data for these genes are not concordant with previously reported findings. According to the literature $C N G B 1, U S H 2 A$, and FSCN2 are all exclusively expressed in photoreceptors, ${ }^{10-14}$ but we obtained more widespread expression profiles. As shown in Figure 2A, the mRNA of rod cGMP-gated channel $\beta$-subunit protein (CNGB1) is detectable in all retinal cell layers both in human and in mouse retinas (Figs. 2A, 2D). A similar result was observed when the $U \boldsymbol{s} \boldsymbol{b}$ erin-2 mRNA (USH2A) was analyzed, as shown in Figures $2 \mathrm{~B}$ and $2 \mathrm{E}$. This pattern was confirmed with two independent probes, one spanning the last exon and the $3^{\prime}$ UTR of the long USH $2 \mathrm{~A}$ isoform ${ }^{15}$ and a second covering the $5^{\prime}$ UTR and the first exon of both the short and the long USH $2 A$ isoforms (see database). Homogeneous expression in human and mouse retina with more intense staining in the ganglion cell layer is also detectable with the RNA probes for the retinal fascin homolog 2 (FSCN2) gene (Figs. 2C, 2F).

A widespread distribution throughout all the layers both in human and mouse retina was also obtained for the Neural retina leucine zipper (NRL) transcript. Four specific probes spanning different regions of the human $N R L$ and two specific probes for the mouse $\mathrm{Nrl}$ transcript showed identical expression patterns (data accessible at http://www.tigem.it/RPexp/ and in Supplementary Fig. S1, http://www.iovs.org/cgi/content/

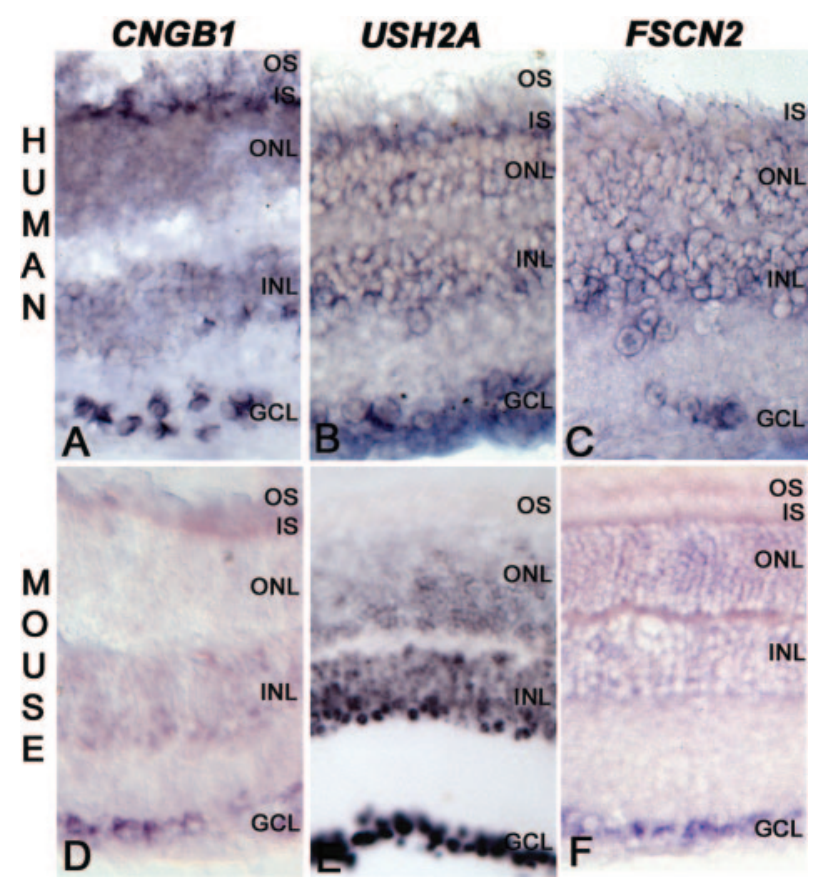

FIGURE 2. Localization of CNGB1, USH2A, and FSCN2 mRNA in human and mouse retinas. Hybridization of human retinas (A-C) and murine retinas (D-F) with RNA probes for: $C N G B 1(\mathbf{A}, \mathbf{D}), U S H 2 A(\mathbf{B}$, E), FSCN2 (C, F). In the human and in the mouse retina, they were widely transcribed in the photoreceptors, INL, and GCL. OS, photoreceptors outer segment; IS, photoreceptors inner segment. 


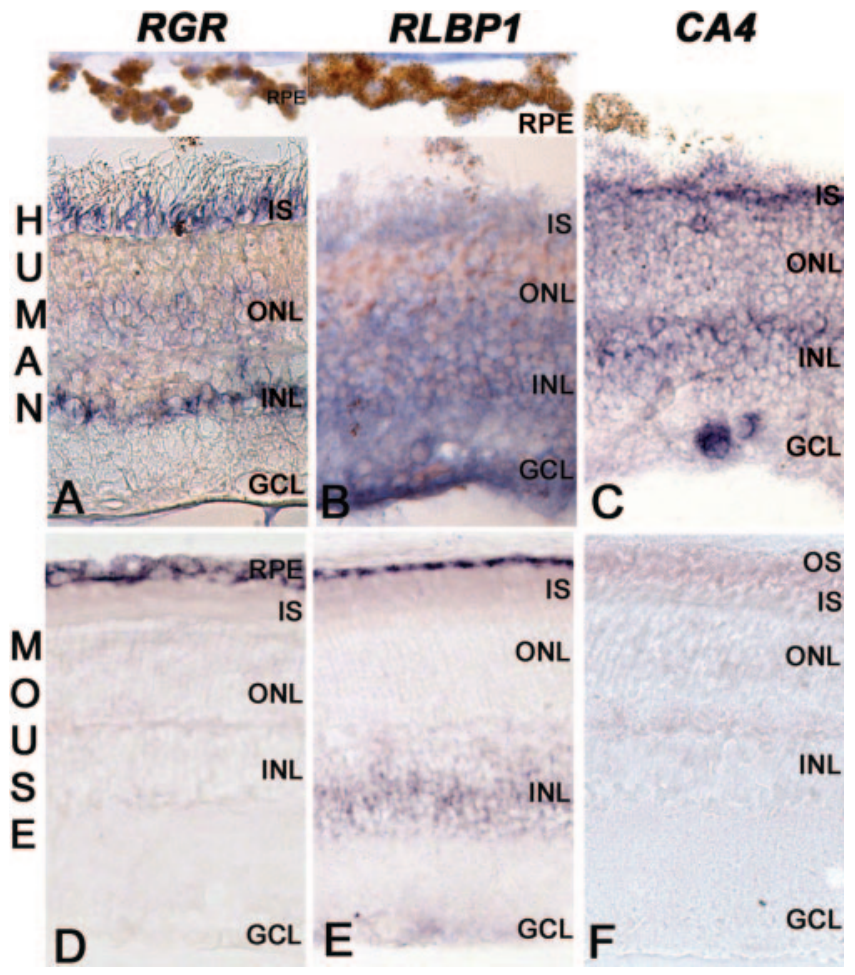

FIGURE 3. Expression profiles of $R G R, R L B P 1$, and $C A 4$ genes. $R G R$ was expressed in the RPE and photoreceptors, and a weak signal was detectable in the INL of the human retina (A). In the mouse retina signal was detectable only in the RPE (D). RLBP1 mRNA was ubiquitously detectable in the human retina and RPE (B), whereas in the mouse retina it was found in the RPE, INL, and weakly in the GCL (E). CA4 mRNA was present in the photoreceptors, INL, and GCL in the human retina (C). No staining was observed in the mouse retina (F). IS, photoreceptors inner segment.

full/49/6/2330/DC1). The expression of the $N r l$ mRNA is also present in the INL and GCL of the $A i p l 1^{-/-}$mouse model, which lacks photoreceptors, ${ }^{9}$ as assessed by the absence of signal after RNA ISH with a rhodopsin probe (Supplementary Fig. S1). A similar expression in the $A i p l 1^{-/-}$retina was also observed with Cngb1-, Ush2A-, and Fscn2-specific RNA probes (data not shown). To test the specificity of the $N r l$ probes used on the mouse eye sections we also performed ISH experiments on P1.5 mouse head sections. As shown in Supplementary Fig. S1H, ISH signal was present, not only in the developing eye but also in other parts of the brain (the olfactory bulb and telencephalon). These results were unexpected, because $\mathrm{Nrl}$ has been described as a rod photoreceptor-specific gene. ${ }^{16-18}$ For these reasons, we decided to investigate $N r l$ mRNA expression by a different method, such as RT-PCR on RNA extracted from the same photoreceptor-deficient mouse $\mathrm{Aipl1}^{-/-}$. $\mathrm{Nrl}$ expression is detectable only in the wild-type retina, whereas no amplification can be observed in Aipl1 $^{-/-}$retina, suggesting that indeed $\mathrm{Nrl}$ is a photoreceptor-specific gene and that ISH is not a proper method for the analysis of the expression of this gene.

We similarly wanted to test the specificity of our ISH data for all the genes whose expression pattern diverged from previously reported data. For this purpose, we performed RTPCR analysis on RNA from 3-month-old $\mathrm{Aipl1^{-/- }}$ and wild-type retinas, with oligonucleotide primers specific for Cngb1, Ush2A, and Fscn2. To confirm the complete photoreceptor degeneration in 3-month-old $\mathrm{Aipl1}^{-/-}$mice, we analyzed, by RT-PCR, expression of a photoreceptor-specific marker (Pde6a) and no expression was detected, as shown in Supplementary Figure S1I. On the other hand, Cngb1, Ush2A, and
Fscn 2 was amplified in both the wild-type and Aipl1 $^{-/-}$retinas, further confirming the expression of these genes outside the photoreceptor cells.

\section{RNA ISH Expression Profiles of the RGR, RLBP1, and $C A 4$ Genes}

We found some notable differences between the expression profiles in human and mouse adult eye sections for the $R G R$, $R L B P 1$, and $C A 4$ genes (Fig. 3). $R G R$ and $R L B P 1$ are members of the visual cycle with reported expression at the protein level in the mouse retina in the RPE; Müller cells; and, in the case of $R L B P 1$, in the ganglion cells. ${ }^{19-22}$ In agreement with previous reports, we observed that in the murine retina both $R g r$ and Rlbp1 mRNAs were expressed in the RPE, with Rlbp1 transcripts also detectable in the INL and in the GCL (Figs. 3D, 3E). Surprisingly, in the human retina, besides the expected expression in the RPE and INL, ${ }^{19,21}$ the $R G R$ transcript was detected also in photoreceptors, and RLBP1 mRNA was distributed in all the different cell types of the retina, including the photoreceptors (Fig. 3B). mRNA localization for each of these genes in human photoreceptors was confirmed with a second, nonoverlapping probe on sections obtained from at least two individuals (see http://www.tigem.it/RPexp/).

We also detected major differences in the expression of the $C A 4$ gene in human and mouse retinas. $C A 4$ is the only gene underlying RP that has been reported not to be expressed in the retina, as the CA4 protein has been shown to be localized in the choriocapillaris surrounding the retina. ${ }^{23,24}$ In our study, two independent $C A 4$ probes labeled all the retinal layers of



Figure 4. RPGR and PAP1 mRNA distribution. The RPGR default variant was expressed in the ONL and INL in the human retina (A), whereas in the mouse retina (D) a faint signal was detected only in the ONL. The RPGR-ORF15 variant showed intense staining in the photoreceptors of the human retina $(B)$, whereas in the mouse retina, the signal was present in the ONL, INL, and GCL (E). PAP1 in the human retina was strongly expressed in the ONL, whereas a weaker expression was also detectable in the INL and GCL (C). On the contrary, in the mouse retina, the signal was prevalent in the GCL and INL, and staining of the ONL was at the limit of detection (F). OS, outer segment. 
the human retinas (Fig. 3C and online database). Conversely, by RNA ISH, we did not detect any signal in the mouse retina (Fig. 3F).

\section{Expression of RPGR and PAP1 Genes in Human Photoreceptors}

Unlike the genes described so far, which displayed significant differences in expression between human and mouse eye sections, RPGR and PAP1 show less evident discrepancy in the retinal expression patterns between these two species. The $R P G R$ gene is characterized by the presence of two main isoforms, a "default variant" that consists of 19 exons and the so-called ORF15 variant, with a terminal exon within intron 15 of the default variant. ${ }^{25}$ To date, the RPGR protein has been shown to be localized in the photoreceptor connecting cilia. ${ }^{25-27}$ However, there are no high-resolution expression data at the RNA level for this gene in either human or mouse retinas and no comparative analysis of the expression pattern of the two main isoforms. We found the RPGR default variant mRNA to be expressed in human photoreceptors and in the INL, whereas in the mouse retina weak staining could be observed only in photoreceptors (Figs. 4A, 4D). The RPGR-ORF15 splice variant was specifically transcribed in human photoreceptors, whereas two independent probes for mouse Rpgr-ORF15 showed mRNA localization in all retinal cells with a more intense staining in the GCL (Figs. $4 \mathrm{~B}, 4 \mathrm{E}$ ).

PAP1 (RP9) is one among the four splicing factors (PRPF3, PRPF31, PRPF8, and PAP1) that have been linked to autosomal dominant RP. ${ }^{28,29}$ There is almost no information available regarding the RNA expression of these splicing factors in the retina. RNA expression patterns for $P R P F 3, P R P F 31$, and PRPF8 obtained in this study suggest that these genes are ubiquitously expressed in all the layers of the retina, with a gradient of expression stronger in the GCL and very low in the photoreceptors, similar to that previously reported for the murine Prpf3 and the human protein ${ }^{30}$ (data available at http:// www.tigem.it/RPexp/). Similar expression was also observed for Pap1 in the mouse retina, where signal intensity in ONL was much lower than in the INL and GCL (Fig. 4F). On the other hand, in human retinas, signal obtained with two specific RNA probes was predominant in the ONL, compared with that in the INL and GCL (Fig. 4C and http://www.tigem.it/RPexp/). It is worth noticing that one of these two probes, 100-bp long, specifically recognizes the PAP1 transcript, whereas the second one may also cross-react with a PAP1 pseudogene. ${ }^{31}$

\section{Discussion}

We generated a high-resolution expression atlas in the human and mouse retina of 34 genes responsible for RP. The generation of this atlas allowed us to infer novel expression data for this biologically relevant group of genes as we report the RNA expression profiles in the retina of 20 human RP genes and of six corresponding murine orthologues that have never been reported before, to the best of our knowledge. In addition, the systematic and comparative expression analysis of all known RP genes may provide new insights into the putative functional activities of some of these genes.

As expected, most of the analyzed genes displayed identical expression patterns in the human and murine eye. It is important to point out that all the genes were analyzed on human eye sections obtained from at least two different individuals, and these results can be retrieved from an ad hoc-generated online database (http://www.tigem.it/RPexp). Comparison of data from two different individuals was crucial because eye bulbs used in this study were obtained from cornea donors with different profiles in terms of age, sex, cause of death, and postmortem time. Analyzing all the genes in different individuals enabled us to verify whether differences in any of the factors pertaining to the donor may interfere with the reliability of the results obtained. That the high similarity in the expression patterns could be observed on eye sections from different donors points out that the tissue-handling procedure was optimal and that the data obtained are reliable.

Within the catalog of the genes with expression patterns conserved between mouse and human, we found three genes for which we observed RNA expression profiles that are not in agreement with previous reports: CNGB1, USH2A, and FSCN2. All three genes have been reported to be expressed only in the photoreceptor layer, ${ }^{10-14}$ whereas our data suggest that they have a more widespread distribution across all retinal cell layers both in humans and in mice. We confirmed this observation by analyzing the expression of these genes in mice lacking photoreceptors (Supplementary Fig. S1, http://www. iovs.org/cgi/content/full/49/6/2330/DC1). The B subunit of the rod cGMP-gated channel (CNGB1), together with subunit A, forms cyclic nucleotide-gated channels that play a critical role in visual transduction. ${ }^{11}$ These two subunits build heteromeric channels that localize in the outer segments of photoreceptors. ${ }^{11,13}$ The CNGB1 protein has been detected not only in photoreceptor cells of the retina but also in olfactory channels, ${ }^{32}$ sperm cells, and other tissues. ${ }^{33}$ In our study, both human and mouse-specific probes show expression of $C N G B 1$ in all the layers of the retina. Hence, it is possible that CNGB1 plays some role in channels assembling in the different neurons of the retina.

The USH2A gene is responsible for both nonsyndromic RP and for Usher syndrome, which is characterized by hearing loss and RP. ${ }^{34}$ USH $2 A$ mRNA expression has been reported exclusively in the retina outer segment in various species, ${ }^{10}$ whereas subsequent studies indicated the presence of USH2A protein in basement membranes and extracellular matrix in various tissues. ${ }^{35,36}$ We used different probes to analyze the two different USH $2 A$ mRNA isoforms to exclude the possibility that the difference that we observed were associated with the different RNA expression patterns of these isoforms, ${ }^{15}$ and we confirmed a diffuse staining in the different retinal layers. Similarly, we also observed ubiquitous expression in both human and mouse of the FSCN2 gene, contrary to previously published photoreceptor-specific expression. ${ }^{12,14}$ Overall, we cannot exclude that the discrepancy between the previously reported photoreceptor-specific localization for the CNGB1, USH2A, and FSCN2 proteins and the ubiquitous mRNA expression obtained in this study is due to a differential distribution of the transcript and the corresponding protein.

Particularly interesting are the findings concerning the $N R L$ gene. NRL (neural retina leucine zipper) causes both autosomal-recessive and -dominant $R P$ and is a retina-specific transcription factor. ${ }^{37} \mathrm{NRL}$ was suggested to be a rod-specific transcription factor, ${ }^{16-18}$ mainly due to the evidence that its inactivation in the mouse causes functional transformation of rods into cones ${ }^{17}$ and that a $\mathrm{Nrl}$-specific promoter drives gene expression specifically in rod photoreceptors in a transgenic mouse. ${ }^{16}$ Nevertheless, data showing restricted expression of $\mathrm{Nrl}$ in photoreceptors (both at the transcript and at the protein levels) are not available in the literature. Our $N R L$ expression data are in agreement with previous reports on RNA and protein expression showing that this gene is expressed in all retinal cell layers, both in different developmental stages and in adults in different species. ${ }^{18,38,39}$ The reliability of the latter data has been subsequently questioned because of the possible nonspecificity of the probes and antibodies used for $N R L$ detection. ${ }^{18}$ In our experiments, we used four independent and highly specific human riboprobes, covering either the 3 '-UTR or alternative $5^{\prime}$ ends of different transcripts belonging to this 
gene. Furthermore, we detected a widespread expression across all mouse retinal cell layers, both on wild-type and Aipl1 ${ }^{-/-}$mouse eye sections when using murine $\mathrm{Nrl}$-specific probes that do not share any sequence identity with other transcribed sequences present in the mouse genome, apart from $N r l$. However, RT-PCR assessment revealed that $N r l$ is expressed only in wild-type mouse retina and not in $\mathrm{Aipl1}^{-/-}$ retina that has lost its photoreceptors, suggesting that indeed this gene is exclusively expressed in photoreceptors. ${ }^{16-18}$ In contrast, Cngb1, Ush2A, and Fscn 2 are expressed both in wild-type and $\mathrm{Aipl1^{-/- }}$ mice, further confirming their expression also in the INL and GCL. We believe that, contrary to all the genes that we have analyzed in this and in previous works, ${ }^{40-42}$ RNA ISH is not the appropriate method for $N R L$ expression analysis. In particular, taking into account the recently reported significant presence in mammalian genomes of noncoding RNAs, ${ }^{43}$ the observed RNA ISH expression of $N R L$ may be explained by the presence of as yet uncharacterized noncoding RNAs transcribed from the NRL genomic locus.

We also detected a significant difference in the expression pattern of the $C A 4$ gene in the human retina compared with that in previous reports. $C A 4$ is an important regulator of $\mathrm{pH}$ balance, because it catalyzes hydration of carbon dioxide. ${ }^{24}$ Human CA4 mRNA was observed in all retina layers, contrary to published protein expression in choriocapillaris. ${ }^{23,24}$ On the other hand, we could not detect the $C A 4$ transcript in the murine retina, perhaps because of the low RNA levels of $C A 4$ in mouse retina or indeed because of a real difference in $C A 4$ localization in the two species.

We detected differences in the expression patterns between human and murine retinas for the following genes: $R G R$, $R L B P 1, R P G R$, and PAP1. RGR and RLBP1 genes are members of the visual cycle cascade, which is responsible for the regeneration of bleached visual pigments. In rods, the visual cycle initiates in the photoreceptor cells, but then it is completed in the RPE. These two genes encode enzymes that are very important for chromophore regeneration in the RPE, ${ }^{20-22}$ although both are also expressed in Müller cells, and RLBP1 is also expressed in the ganglion cells. ${ }^{19,22}$ We confirmed the expected expression pattern in the RPE of $R g r$ and Rlbp1 genes and Rlbp1 expression in the INL and GCL in the mouse eye. However, in the human retina we observed RGR mRNA, not only in the RPE and INL but also in the ONL. Similarly, $R L B P 1$ is expressed in RPE and also in the ONL, INL, and GCL. The function of these two genes is well established in the $\mathrm{RPE}^{20-22}$; however, their exact role in the cells others than RPE is still not clear. The newly reported RNA expression domains of $R G R$ and $R L B P 1$ in photoreceptors in humans are worthy of further investigation for a better understanding of their function outside the RPE.

The comparative RNA ISH analysis of RP genes in human and mouse retinas also revealed interesting expression patterns for the RPGR and PAP1 genes. RPGR (retinitis pigmentosa GTPase regulator) is necessary for maintenance of photoreceptor viability. ${ }^{25}$ Two splice variants are present in the retina: the default form and the so-called ORF15 form, which harbors a mutation hot spot for RP. ${ }^{27,44}$ We performed RNA ISH analysis with probes specifically recognizing each of the two forms in human and murine retina and found different expression profiles. Both RPGR proteins were previously detected in photoreceptor outer segments. ${ }^{25}$ Of note, ORF15 shows a specific expression in the human photoreceptors that are the main targets of RP, whereas the default form, although predominantly expressed in photoreceptors, can be detected also in the INL. The photoreceptor-specific transcription of $R P G R$ ORF15 correlates nicely with the observation that most RPGR mutations that cause the RP phenotype fall within this particular splice variant. Likewise, $P A P 1$ is also predominantly ex- pressed in photoreceptors in the human retina compared with mouse retina, where it is expressed mainly in the GCL and INL. $P A P 1$, as well as PRPF3, PRPF31, and PRP8, is a pre-mRNA splicing factor. ${ }^{29,45-47}$ It is very challenging to understand why mutations in ubiquitously expressed splicing factors cause such a specific phenotype as RP. Our expression analysis of PRPF3, PRPF31, and PRPF8 did not show abundant expression in photoreceptors. Only PAP1 mRNA appears more abundant in human photoreceptors compared with other retinal cells. These observations may open new perspectives for the study of the link between pre-mRNA splicing factors and RP pathogenesis.

This study provides the first systematic comparative expression analysis in the human and in the mouse retina of all known genes responsible for RP (RP gene expression atlas, http:// www.tigem.it/RPexp/). We believe that our study gives important insights into the function of these genes by providing essential gene expression data in the human retina. The differences observed in the expression patterns of some of the analyzed genes in humans and mice may shed new light on the function of these genes and their roles in disease pathogenesis and will be a fundamental support in the correct transfer of information obtained from animal models to patients with RP.

\section{Acknowledgments}

The authors thank Giampiero Lago for help in the design of the Web site, Giovanna Alfano for providing reagents and for helpful discussion, and Enrico Maria Surace and Simona Neglia for providing $\mathrm{Aipl1}^{-/-}$ mouse eye sections.

\section{References}

1. Farrar GJ, Kenna PF, Humphries P. On the genetics of retinitis pigmentosa and on mutation-independent approaches to therapeutic intervention. EMBO J. 2002;21:857-864.

2. Hims MM, Diager SP, Inglehearn CF. Retinitis pigmentosa: genes, proteins and prospects. Dev Ophthalmol. 2003;37:109-125.

3. Phelan JK, Bok D. A brief review of retinitis pigmentosa and the identified retinitis pigmentosa genes. Mol Vis. 2000;6:116-124.

4. Dalke C, Graw J. Mouse mutants as models for congenital retinal disorders. Exp Eye Res. 2005;81:503-512.

5. Maronpot R, Boorman, GA, Gaul, BW. Pathology of the Mouse. Vienna, IL: Cache River Press;1999;471-480.

6. Aherne A, Kennan A, Kenna PF, et al. On the molecular pathology of neurodegeneration in IMPDH1-based retinitis pigmentosa. Hum Mol Genet. 2004;13:641-650.

7. Buniello A, Montanaro D, Volinia S, Gasparini P, Marigo V. An expression atlas of connexin genes in the mouse. Genomics. 2004;83:812-820.

8. Reymond A, Marigo V, Yaylaoglu MB, et al. Human chromosome 21 gene expression atlas in the mouse. Nature. 2002;420:582-586.

9. Dyer MA, Donovan SL, Zhang J, et al. Retinal degeneration in Aipl1-deficient mice: a new genetic model of Leber congenital amaurosis. Brain Res. 2004;132:208-220.

10. Huang D, Eudy JD, Uzvolgyi E, et al. Identification of the mouse and rat orthologs of the gene mutated in Usher syndrome type IIA and the cellular source of USH2A mRNA in retina, a target tissue of the disease. Genomics. 2002;80:195-203.

11. Huttl S, Michalakis S, Seeliger M, et al. Impaired channel targeting and retinal degeneration in mice lacking the cyclic nucleotidegated channel subunit CNGB1. J Neurosci. 2005;25:130-138.

12. Saishin $\mathrm{Y}$, Shimada $\mathrm{S}$, Morimura $\mathrm{H}$, et al. Isolation of a cDNA encoding a photoreceptor cell-specific actin-bundling protein: retinal fascin. FEBS Lett. 1997;414:381-386.

13. Trudeau MC, Zagotta WN. An intersubunit interaction regulates trafficking of rod cyclic nucleotide-gated channels and is disrupted in an inherited form of blindness. Neuron. 2002;34:197-207.

14. Yokokura S, Wada Y, Nakai S, et al. Targeted disruption of FSCN2 gene induces retinopathy in mice. Invest Ophthalmol Vis Sci. 2005; $46: 2905-2915$ 
15. van Wijk E, Pennings RJ, te Brinke $\mathrm{H}$, et al. Identification of 51 novel exons of the Usher syndrome type 2A (USH2A) gene that encode multiple conserved functional domains and that are mutated in patients with Usher syndrome type II. Am J Hum Genet. 2004;74:738-744.

16. Akimoto M, Cheng $\mathrm{H}$, Zhu D, et al. Targeting of GFP to newborn rods by $\mathrm{Nrl}$ promoter and temporal expression profiling of flowsorted photoreceptors. Proc Natl Acad Sci USA. 2006;103:3890 3895.

17. Mears AJ, Kondo M, Swain PK, et al. Nrl is required for rod photoreceptor development. Nat Genet. 2001;29:447-452.

18. Swain PK, Hicks D, Mears AJ, et al. Multiple phosphorylated isoforms of NRL are expressed in rod photoreceptors. J Biol Chem. 2001;276:36824-36830.

19. Bailey MJ, Cassone VM. Opsin photoisomerases in the chick retina and pineal gland: characterization, localization, and circadian regulation. Invest Opbthalmol Vis Sci. 2004;45:769-775.

20. Bunt-Milam AH, Saari JC. Immunocytochemical localization of two retinoid-binding proteins in vertebrate retina. J Cell Biol. 1983;97: 703-712.

21. Fong HK, Lin MY, Pandey S. Exon-skipping variant of RGR opsin in human retina and pigment epithelium. Exp Eye Res. 2006;83:133140 .

22. Kennedy BN, Li C, Ortego J, Coca-Prados M, Sarthy VP, Crabb JW CRALBP transcriptional regulation in ciliary epithelial, retinal Muller and retinal pigment epithelial cells. Exp Eye Res. 2003;76:257260.

23. Nagelhus EA, Mathiisen TM, Bateman AC, et al. Carbonic anhydrase XIV is enriched in specific membrane domains of retinal pigment epithelium, Muller cells, and astrocytes. Proc Natl Acad Sci USA. 2005;102:8030-8035.

24. Yang Z, Alvarez BV, Chakarova C, et al. Mutant carbonic anhydrase 4 impairs $\mathrm{pH}$ regulation and causes retinal photoreceptor degeneration. Hum Mol Genet. 2005;14:255-265.

25. Hong DH, Pawlyk B, Sokolov M, et al. RPGR isoforms in photoreceptor connecting cilia and the transitional zone of motile cilia. Invest Opbthalmol Vis Sci. 2003;44:2413-2421.

26. Hong DH, Yue G, Adamian M, Li T. Retinitis pigmentosa GTPase regulator (RPGRr)-interacting protein is stably associated with the photoreceptor ciliary axoneme and anchors RPGR to the connecting cilium. J Biol Chem. 2001;276:12091-12099.

27. Shu X, Black GC, Rice JM, et al. RPGR mutation analysis and disease: an update. Hum Mutat. 2007;28:322-328.

28. Inglehearn CF, Carter SA, Keen TJ, et al. A new locus for autosomal dominant retinitis pigmentosa on chromosome 7p. Nat Genet. $1993 ; 4: 51-53$.

29. Keen TJ, Hims MM, McKie AB, et al. Mutations in a protein target of the Pim-1 kinase associated with the RP9 form of autosomal dominant retinitis pigmentosa. Eur J Hum Genet. 2002;10:245249.

30. Comitato A, Spampanato C, Chakarova C, Sanges D, Bhattacharya SS, Marigo V. Mutations in splicing factor PRPF3, causing retinal degeneration, form detrimental aggregates in photoreceptor cells. Hum Mol Genet. 2007;16:1699-1707.

31. Bischof JM, Chiang AP, Scheetz TE, et al. Genome-wide identification of pseudogenes capable of disease-causing gene conversion. Hum Mutat. 2006;27:545-552.
32. Sautter A, Zong X, Hofmann F, Biel M. An isoform of the rod photoreceptor cyclic nucleotide-gated channel beta subunit expressed in olfactory neurons. Proc Natl Acad Sci USA. 1998;95: 4696 - 4701 .

33. Biel M, Zong X, Ludwig A, Sautter A, Hofmann F. Molecular cloning and expression of the modulatory subunit of the cyclic nucleotide-gated cation channel. J Biol Chem. 1996;271:63496355.

34. Kremer H, van Wijk E, Marker T, Wolfrum U, Roepman R. Usher syndrome: molecular links of pathogenesis, proteins and pathways: human molecular genetics. 2006;15:R262-R270.

35. Pearsall N, Bhattacharya G, Wisecarver J, Adams J, Cosgrove D, Kimberling W. Usherin expression is highly conserved in mouse and human tissues. Hear Res. 2002;174:55-63.

36. Reiners J, Nagel-Wolfrum K, Jurgens K, Marker T, Wolfrum U. Molecular basis of human Usher syndrome: deciphering the meshes of the Usher protein network provides insights into the pathomechanisms of the Usher disease. Exp Eye Res. 2006;83:97119

37. Swaroop A, Xu JZ, Pawar H, Jackson A, Skolnick C, Agarwal N. A conserved retina-specific gene encodes a basic motif/leucine zipper domain. Proc Natl Acad Sci USA. 1992;89:266-270.

38. He L, Campbell ML, Srivastava D, et al. Spatial and temporal expression of AP-1 responsive rod photoreceptor genes and bZIP transcription factors during development of the rat retina. Mol Vis. 1998;4:32.

39. Yoshida S, Mears AJ, Friedman JS, et al. Expression profiling of the developing and mature $\mathrm{Nrl}^{-/-}$mouse retina: identification of retinal disease candidates and transcriptional regulatory targets of Nrl. Hum Mol Genet. 2004;13:1487-1503.

40. Boguski MS, Tolstoshev CM, Bassett DE, Jr. Gene discovery in dbEST. Science. 1994;265:1993-1994.

41. Karali M, Peluso I, Marigo V, Banfi S. Identification and characterization of microRNAs expressed in the mouse eye. Invest Opbthalmol Vis Sci. 2007;48:509-515.

42. Redmond TM, Yu S, Lee E, et al. Rpe65 is necessary for production of 11-cis-vitamin A in the retinal visual cycle. Nat Genet. 1998;20: $344-351$

43. Kapranov P, Cheng J, Dike S, et al. RNA maps reveal new RNA classes and a possible function for pervasive transcription. Science. 2007;316:1484-1488.

44. Vervoort R, Lennon A, Bird AC, et al. Mutational hot spot within a new RPGR exon in X-linked retinitis pigmentosa. Nat Genet. 2000; 25:462- 466

45. Chakarova CF, Hims MM, Bolz H, et al. Mutations in HPRP3, a third member of pre-mRNA splicing factor genes, implicated in autosomal dominant retinitis pigmentosa. Hum Mol Genet. 2002;11:8792.

46. McKie AB, McHale JC, Keen TJ, et al. Mutations in the pre-mRNA splicing factor gene PRPC8 in autosomal dominant retinitis pigmentosa (RP13). Hum Mol Genet. 2001;10:1555-1562.

47. Vithana EN, Abu-Safieh L, Allen MJ, et al. A human homolog of yeast pre-mRNA splicing gene, PRP31, underlies autosomal dominant retinitis pigmentosa on chromosome 19q13.4 (RP11). Mol Cell. 2001;8:375-381. 